\title{
Tangence
}

\section{Les cauchemars d'Albert Laberge}

\section{Denis Sauvé}

Numéro 50, mars 1996

Lectures de nouvelles québécoises

URI : https://id.erudit.org/iderudit/025896ar

DOI : https://doi.org/10.7202/025896ar

Aller au sommaire du numéro

Éditeur(s)

Tangence

ISSN

0226-9554 (imprimé)

1710-0305 (numérique)

Découvrir la revue

Citer cet article

Sauvé, D. (1996). Les cauchemars d'Albert Laberge. Tangence, (50), 118-130.

https://doi.org/10.7202/025896ar d'utilisation que vous pouvez consulter en ligne.

https://apropos.erudit.org/fr/usagers/politique-dutilisation/ 


\section{Les cauchemars d'Albert Laberge Denis Sauvé}

Le bonheur c'est, lorsqu'on est fatigué, une brève halte sous de grands ormes ombreux, mais le sol est couvert de larges bouses de vaches; c'est, lorsqu'on a soif, un gobelet d'eau frầche et limpide, mais à la surface du puits, flotte le corps enflé d'un chien noyé.

AlbFrt LABERGE, Le destin des bommes

\section{L'auteur et le personnage}

Nous connaissons Albert Laberge grâce à la réédition posthume de La Scouine ${ }^{1}$ et à l'Anthologie d'Albert Laberge ${ }^{2}$ assemblée par Gérard Bessette. Ces deux livres ont sauvé de l'oubli un écrivain qui s'excluait lui-même du champ littéraire, car Laberge maintenait une posture pour le moins paradoxale face à l'édition. Il édite ses livres à compte d'auteur, tirés à soixante-quinze ou cent exemplaires, en donne quelques-uns à des amis et accumule les autres dans son grenier. Il refuse toute diffusion commerciale de ses volumes, mais il fait paraître dix-huit extraits de La Scouine et une cinquantaine de nouvelles dans dix périodiques différents, dont La Presse, La Semaine et La Patrie.

S'il publie des nouvelles dans les journaux, il ne craint ni la condamnation ni l'oubli. Comment, alors, expliquer son refus de diffuser sur le marché culturel une imposante ouvre comportant pas moins de treize volumes imprimés, dont sept recueils de nouvelles? Le livre, objet par excellence du culte littéraire, souvent associé au mythe de la pérennité, semble apparaître aux yeux de Laberge comme une menace à laquelle échapperaient les éphé-

1 Albert Laberge, La Scouine, Montréal, L'Actuelle, 1972, 134 p. Voir aussi l'édition critique établie par Paul Wyczynski, Montréal, Les Presses de l'Université de Montréal, coll. * Bibliothèque du Nouveau Monde*, 1986, 297 p.

2 Gérard Bessette, Anthologie d'Albert Labenge, Ottawa, Le Cercle du Livre de France, $1962,310 \mathrm{p}$. 
mères périodiques. Que penser de la fausse modestie de ses propres justifications? "[Cle qui compte, c'est la joie d'écrire, de produire une œuvre, d'exprimer ce qu'il y a en soi. Qu'importe après cela que le livre soit imprimé ou non? L'auteur a eu la somme de satisfaction qu'il pouvait espérer. " ${ }^{3}$ De toute évidence, un noud conscient ou inconscient empêchait Laberge d'abandonner ses livres sur les rayons des librairies pour qu'ils soient vendus, lus, critiqués - encensés ou oubliés. Peut-être voulait-il préserver un fantasme de gloire en refusant de subir l'épreuve de réalité. Publiant ses volumes en édition privée et hors commerce, Laberge ne protégeait-il pas son fragile narcissisme d'écrivain contre tout discours de dévalorisation qu'auraient pu émettre les critiques de son époque? Puisque l'altérité correspondait à un danger (de condamnation, de déni, de castration), il érigeait son propre culte en circuit fermé, rêvant peut-être d'une consécration posthume. De fait, c'est en 1963, trois ans après sa mort, que surgissent pour la première fois sur les rayons des librairies quelques-uns de ses textes, réunis dans l'Antbologie d'Albert Laberge.

Gérard Bessette commence sa préface par ces lignes: "Je tiens à l'affirmer dès le début: à mon avis Albert Laberge est de beaucoup notre plus grand nouvelliste, le seul qui atteigne parfois à la puissance d'un Maupassant, d'un Zola. " "Grâce à sa biographie sommaire, à ses citations de témoignages élogieux et à sa préface apologétique, cette anthologie constitue une véritable amorce de consécration. Par la suite, en 1971, La Scouine est réédité et, depuis, un certain nombre de mémoires, de thèses, d'articles et de monographies ont été produits sur l'œuvre de Laberge, mais on attend toujours la réédition de ses recueils.

\section{Naturalisme et postulats interprétatifs}

Albert Laberge est généralement classé parmi les écrivains naturalistes; ainsi la critique tend-elle à interpréter spontanément ses récits en les naturalisant. L'étiquette programme donc l'interprétation de telle sorte que lorsque Jacques Brunet parle de

\footnotetext{
3 Albert Laberge, Joumalistes, écrivains et artistes, Montréal, Édition privée, 1945, p. 145-146, cité par Jacques Brunet, Albert Labenge: sa vie et son cuuve, Ottawa, Éditions de l'Université d'Ottawa, 1969, p. 24.

4 Gérard Bessette, op. cit., p. VII.
} 
"pessimisme outranciern" ${ }^{5}$, lorsque Bessette emploie les termes "noircissement systématique de la réalité "6, lorsque Samiha Yassa-Gad inclut des statistiques et des extraits de documents sociologiques dans son mémoire sur Laberge ${ }^{7}$, on semble oublier que la littérature naturaliste reste, après tout, de la littérature. Comme l'écrit Claude Lafarge, la présumée représentation du réel, dans les textes narratifs, fait d'abord référence à un univers de fiction:

Toute représentation fictive procède d'une convention littéraire, appliquée ou niée: l'univers de référence des fictions est d'abord un univers de fictions constituées et consacrées par l'usage ou les autorités, ce qu'on peut appeler le réel fictif. Ceci ne veut nullement dire que ces représentations sont fausses par nature, mais que l'appréciation que peut en avoir le consommateur dépend avant tout de cette référence $[\ldots]^{8}$

Que reproche-t-on vraiment à l'univers de Laberge? Jacques Brunet, constatant qu'»[on] n'en finirait pas d'énumérer toutes les cruautés du destin envers les personnages" "dans les nouvelles labergiennes, y voit le déploiement d'une fatalité gratuite et conclut que l'auteur "déforme systématiquement et - disons le mot - un peu bêtement la réalité *10. Gérard Bessette, qui applique un jugement similaire en le limitant à La Scouine, affirme que ce roman (dont il n'inclut que six chapitres dans son anthologie) paraît invraisemblable parce qu'il manque d'، explications [...] [sur les] causes particulières : historiques, sociales, héréditaires, économiques, etc." ${ }^{11}$ entraînant le malheur des personnages; cette absence d' "explications " semble justifier pour Bessette un jugement de noircissement systématique de la réalité.

La critique naturalise l'écriture labergienne dans la mesure où elle adhère naïvement à la prétention du naturalisme à reproduire la réalité, à réussir une mimésis. En regard de cette prétention, l'œuvre de Laberge, ainsi que ceux de nombreux écrivains

\footnotetext{
5 Ibid., P. 79

6 Gérard Bessette, op. cit., p. X.

7 Samiha Yassa-Gad, *Les enfants dans l'œuvre d'Alber Laberge •, mémoire de maîtrise, Montréal, Université McGill, février 1983, 138 f.

8 Claude Lafarge, La valeur littéraire. Figuration littéraire et usages sociaux des fictions, Paris, Fayard, 1983, p. 285.

9 Jacques Brunet, op. cit., p. 79.

10 Ibid., p. 79

11 Ibid, p. XI.
} 
naturalistes, est perçu comme invraisemblable, comme un échec du projet réaliste, puisqu'il se complaît à ne "décrire" que les aspects désagréables de la *réalitén. Par contre, si nous observons les nouvelles en faisant abstraction de l'illusoire visée naturaliste, du projet explicite de Laberge de représenter la réalité ${ }^{12}$, nous ne verrons plus un échec de la mimésis, mais une réussite de la diêgèse, dans la création d'un univers à la fois vraisemblable et absolument invraisemblable. La plume de Laberge fabrique admirablement des cauchemars d'autant plus affreux qu'ils sont parsemés d'effets de réel foudroyants. Sa complaisance dans la description des zones les plus hideuses et les plus douloureuses de la "réalité - d'une réalité onirique savamment déguisée - correspond à la fusion d'un regard lucide mais limité sur le monde, et de la projection sur un écran interminablement noir d'une horde de monstres intimes.

\section{L'effet de recueil}

Nous ne pourrions reprocher aux recueils de Laberge de manquer de cohérence. Au contraire, les multiples sévices qui pulvérisent l'un après l'autre la presque totalité des personnages produisent inévitablement un sentiment de prévisibilité générale des nouvelles que nous pourrions généraliser par un énoncé comme celui-ci : le personnage labergien est destiné à souffrir, à faire souffrir d'autres personnages, à profiter d'eux, et ses désirs, aussi médiocres soient-ils, ne sont qu'exceptionnellement satisfaits. Malédiction, égoïsme, cruauté, mesquinerie, aveuglement, douleur, atrocité, calamité; la sordidité est à peu près sans limites dans l'univers labergien. Souvent, le narrateur exprime ouvertement son dégoût, son mépris ou, au mieux, sa pitié pour les personnages qu'il torture inlassablement. "L'homme de Laberge est presque toujours déterminé par la misère sociale, la souffrance physique, la fatalité 13 , écrit Paul Wyczynski; convaincu que l'auteur ne

12 «Dans mes contes, j'ai voulu être le peintre de la vie, la vie avec ses tragédies, ses drames, ses trahisons, ses fourberies, ses cruelles désillusions. Lá vie a été mon modèle, la pauvre vie. Albert Laberge, lettre à $M$. Lebel, cité par Gabrielle Clerc, - La vision du monde d'Albert Laberge *, mémoire de maîtrise, Québec Université Laval, 1961, f. 42.

13 Paul Wyczyski, - Introduction*, dans Albert Laberge, La Scouine, Montréal, Les Presses de l'Université de Montréal, coll. * Bibliothèque du Nouveau Monde *, 1986, p. 21. 
cherche qu'à "choisir dans la vie paysanne ce qui est tristesse, maladie, souffrance, bêtise humaine, bref la vie sous le signe d'un pessimisme forcé, sous le poids de la fatalité la plus totale "14. Jean-Pierre Boucher ajoute que le personnage de Laberge est prisonnier d'un destin circulaire: "Toujours il est ramené à son point de départ, jamais il n'arrive à sortir du rang, à émerger de l'interminable procession d'aveugles que forment les humains en marche vers le trou dans la terre où s'achève la vie de chacun. "15

Quiconque a lu les recueils publiés par Laberge peut constater comment la presque totalité des récits semble traversée par une pulsion de mort, qui se manifeste tantôt directement - par les morts, maladies, meurtres, viols, trahisons et revers du destin - et tantôt indirectement - par le mépris ou le dédain affiché par le narrateur, les métaphores et les comparaisons répugnantes, etc. L'univers labergien, dominé par un imaginaire sadique, révèle un déterminisme impitoyable qui, saisissant à la réception de nombreuses nouvelles, peut perdre son impact à la lecture successive des nouvelles. Jacques Brunet décrit bien ce processus en affirmant que *[c]'est l'accumulation de ces coïncidences malheureuses, nouvelle après nouvelle, qui lasse le lecteur et donne une impression d'artificiel, de mécanique ${ }^{16}$. Plus loin, il ira même jusqu'à dire qu'en raison de cette fatalité générale, Laberge "ne peut survivre que dans les anthologies" "17.

Bessette se met à l'abri de tels commentaires en présentant une anthologie équilibrée dans laquelle figurent quelquès nouvelles parmi les moins cauchemardesques signées par cet auteur. Il peut donc écrire que: "Du tragique pur au comique narquois en passant par deux intermédiaires, [Laberge] exploite la gamme entière de son clavier. "18 Peu de gens viendront le contredire, puisque son anthologie demeure le seul recueil de nouvelles labergiennes à grand tirage. Par contre, une lecture des recueils originaux permet de constater à quel point Laberge a usé d'une petite portion de son "clavier" et que son écriture effleure rarement le "comique narquois" ainsi que les "intermédiaires". En

14 Ibid., p. 44.

15 Jean-Pierre Boucher, Le recueil de nouvelles. Études sur un genre littéraire dit mineur, Montréal, Fides, 1992, p. 182.

16 Jacques Brunet, op. cit., p. 79.

17 Ibid., p. 85.

18 Gérard Bessette, op. cit., p. XXIII. 
général, l'esthétique des recueils auto-édités par Laberge correspond mieux à l'univers de La Scouine qu'à la section "nouvelles * de l'anthologie de Bessette. Dans ces recueils, généralement sombres, s'entremêlent un pathos ténébreux et un humour noir des plus amers, tandis que l'anthologie offre une plus grande part d'anecdotisme, de grivoiserie, de comique et de légèreté.

\section{Deux cas d'espèce}

Pourquoi Bessette a-t-il divisé la section de son anthologie consacrée aux nouvelles en deux parties intitulées respectivement "La campagne" et " La ville"? Il ne semble pas exister d'opposition fondamentale chez Laberge entre ville et campagne; ces deux milieux paraissent contigus et les personnages se déplacent de l'un à l'autre sans qu'il y ait de changement majeur dans la cruauté de leur destin. Tout au plus constatons-nous quelques variantes inévitables liées à la matérialité de ces lieux ou à leur économie respective. En fait, il serait peut-être plus pertinent de départager les nouvelles labergiennes selon des contrastes plus significatifs que la dualité ville-campagne. Par exemple les deux nouvelles que nous commenterons divergent par leurs visées esthétiques. "Il marie sa fille " 19 appartient au versant "comique " de l'univers labergien, même si l'humour noir de cette nouvelle a un arrière-goût macabre, tandis que "Les noces d'or " 20 offre une structure dramatique.

\section{“ Il marie sa fille "}

Le mariage, dans cette nouvelle, correspond à une fraude magistrale. Isodore Tamareau, constatant que sa fille Liose va mourir de la tuberculose, veut la marier pour ne pas payer l'enterrement. Il repère rapidement sa victime en le personnage de Pilon, un jeune homme *timide, gêné, sans volonté, incapable de dire non [...] Sous divers prétextes, les camarades lui empruntaient de l'argent et ne rendaient jamais la somme complète [...] Une bonne poire.» (p. 158). Cependant, la fille de Tamareau n'est pas suffisamment attirante.

19 Ibid., p. 156-166. Toutes les citations ultérieures d'Albert Laberge renverront aux pages de cette édition. 
124

Elle était bien pâle, bien maigre, bien abattue, pas bien tentante, à vrai dire, pas clu tout ce qu'il faut pour attirer un garçon. Ah! oui, il lui faudrait un stimulant. Il pensait à ça, Tamareau, parce que son père avait été un fameux maquignon qui savait doper un cheval pour lui donner de l'allure, le rendre fringuant. Combien de fois n'avait-il pas vu le vieux administrer de formidables doses de drogue à de vieilles cavales, à de lamentables haridelles qui donnaient pour le moment le change à l'acheteur. (p. 159-160)

Dès lors, la fille est abondamment fardée et droguée par celui que la narrateur a désigné comme "un bon père" (p. 156) et qui ne rêve que d'acheter "une automobile dans laquelle un honnête homme part le dimanche pour se reposer de son travail" (p. 157). Au jour du mariage, droguée plus que jamais, Liose s'évanouit; elle s'éteint quelques jours plus tard et le jeune marié, qui doit emprunter à un usurier pour payer l'enterrement, "est pris dans un terrible engrenage. Il n'en sortira peut-être jamais (p. 166).

Ici, l'humour noir s'appuie sur le style ironique et sur une chosification des victimes qui sont réduites à leur valeur transactionnelle. Pilon, la "bonne poire", n'a aucune volonté et sa fonction au sein du récit consiste à tomber passivement dans le piège de Tamareau; Liose est un personnage muet - équivalent à un cheval malade - que l'on vend à un imbécile pour s'acheter une automobile, sa fonction consiste à être droguée, vendue, et jamais le texte ne fait référence à sa souffrance, sauf à la dernière page lorsqu'elle pense à ses sœurs mortes, victimes elles aussi de la tuberculose:

Liose n'était plus qu'une loque. Elle se sentait si mal qu'elle dut garder le lit. On lui avait cléclaré qu'elle prendrait du mieux, mais maintenant, elle commençait à comprendre que ses espoirs étaient des illusions. Avec découragement, elle songeait à ses soeurs parties si jeunes et elle réalisait qu'elle aurait le même sort. (p. 166)

"Il márie sa fille" est une des rares nouvelles de Laberge dans laquelle un personnage réalise ses désirs, où la volonté ne mène pas à l'échec. Les nouvelles qui représentent de telles réussites individuelles, de tels personnages triomphants, même si elles ne participent pas toujours de l'humour noir, comportent toutes ce caractère fondamental: le bonheur d'un être s'appuie sur la destruction d'un autre, le plaisir est essentiellement égoïste et le personnage qui réalise son ambition doit être cruel ou indifférent 
aux autres. Le plan de Tamareau est parfaitement exécuté aux dépens de deux victimes, l'une morte et l'autre absorbée dans l'engrenage de l'endettement :

Et, le dimanche matin, pendant qu'engoncé dans son habit de deuil acheté à crédit, [Pilon] s'en va à la messe en songeant aux échéances qui arrivent si vite, aux lourds intérêts qu'il paie aux prêteurs juifs, monsieur Isoclore Tamareau part d'un cour léger avec sa femme dans leur auto neuve toute reluisante et se clirige vers les campagnes verdoyantes que traversent des rivières miroitantes au soleil. Ce sont d'honnêtes bourgeois qui prennent un peu de distraction après le travail de la semaine. (p. 166)

\section{«Les noces d'or»}

Julien Mattier va fêter ses cinquante années de mariage avec Amanda Level. Pour l'occasion, il invite ses trois enfants dans l'espoir qu'ils lui donneront de riches cadeaux. Il est pauvre, endetté par une triple hypothèque contractée sur sa ferme; il a toujours eu peur de dépenser et a exploité ses enfants jusqu'à ce que ceux-là se révoltent. La veille des noces d'or, il reçoit une missive exigeant le paiement d'une dette et, comme il ne peut la rembourser, il se voit au bord de la faillite. Lorsque ses enfants surviennent et lui donnent de modestes présents, il les accable de reproches et ceux-là, révoltés, partent à jamais.

Dans cette nouvelle, famille et société sont régies par la seule loi de l'exploitation. Les rapports entre les êtres et les choses, principalement transactionnels, sont déterminés par la valeur marchande et excluent d'avance toute liberté, tout désintéressement, tout don de soi ou tout progrès; sexualité, travail, argent, nourriture et cadeaux correspondent à de la monnaie que l'on s'arrache les uns aux autres, causant souffrance et désagrégement.

Dès la première page, le narrateur déploie cette logique de parasitisme. Puisque ses voisins ont reçu de somptueux présents à l'occasion de leurs noces d'or, Julien Mattier pense qu'il "serait opportun" de fêter les siennes. L'appât du gain, moteur central des comportements de Mattier dans ce récit, va de pair avec l'interminable dépossession qui ruine tous les personnages. Dès qu'il a pris sa décision, il se rend au restaurant où travaille sa fille Mélanie pour l'inviter. La description du repas se limite à ces lignes: 
126

Chaque fois qu'il allait en ville vendre ses produits au marché, il allait la voir, histoire de dîner sans bourse délier. Il mangeait, puis :

- Ma fille paiera, disait-il au patron.

Il avait toujours agi ainsi. Toute sa vie il avait exploité ses enfants. (p. 39)

Puisque Julien Mattier a reçu un maigre héritage de son père, il a dû emprunter pour acheter sa terre, et la peur de ne pouvoir payer l'hypothèque l'a rendu si avare qu'il craint d'être dépossédé par ses pairs: "Lorsqu'on était à table: il regardait chaque bouchée que ses enfants avalaient et ses regards étaient un reproche muet" (p. 41). Lorsque la dette sera remboursée, promet-il un jour à sa famille, il fera bâtir une maison en briques rouges ( avec des chambres en haut" - p. 41); mais même dans cet espoir, l'égoïsme isole les êtres, car chacun ne désire la maison que pour soi. Il suffit qu'une des filles imagine sa future chambre pour que la mère se révolte vivement contre celle qui salit sa maison chimérique :

- Descends de la chambre d'en haut! Que je ne te voie pas clans la chambre d'en haut! criait-elle, fâchée, à Mélanie.

Ces imaginaires chambres d'en haut, c'était son salon. Il ne fallait pas y entrer, y mettre les pieds. (p. 42)

Dans un long paragraphe, l'hypothèque est comparée à une maladie vénérienne dont le progrès morbide se révèle irréversible. Plus loin, la dette devient une charrette que l'on doit faire avancer vers le sommet d'une pente, mais qui recule sans cesse malgré les efforts du cheval et l'assistance de la famille entière. De fait, la dette originelle, qui ne sera jamais surmontée, semble un virus qui contamine la cellule familiale. Le père trouve un emploi à chacun de ses enfants pour leur soutirer l'ensemble de leur salaire. Mais l'exploitation dépasse vite le cadre familial. Emma, placée par son père dans un restaurant, vend bientôt son corps dans un bordel tandis que Mélanie, pratiquement violée dès sa première journée de travail, arrête de donner son salaire de cuisinière au père pour le verser à "l'un des habitués de la gargote * (p. 44). Rosalie, abandonnée par son mari, devient chambreuse et vit "en concubinage avec un parasite" (p. 50). Le fils quitte la terre, devient voleur, fait un séjour en prison et redevient voleur... Tous cessent de payer le père pour entrer dans une nouvelle aliénation: la putain loue son corps, la cuisinière et 
la chambreuse entretiennent chacune un homme, le voleur n'est qu'en sursis de liberté. Il n'y a d'échanges humains que dans la transaction, le vol, le viol, l'exploitation. Bientôt, les enfants cessent de jouer les bêtes de somme: "les filles avaient cessé d'être les dociles et patientes pourvoyeuses de leur père. Elles avaient pratiquement fini de lui donner un revenu. À la fin, elles avaient secoué le joug, et il n'en tirait presque plus rien" (p. 44). Elles ne sont plus attelées au fardeau de la dette, mais ne trouvent aucune liberté. Mélanie, par exemple, se fait soutirer son salaire par un "mâle rapace et fainéant. Après avoir toujours donné tout son argent à son père, elle trouvait naturel de le remettre à ce vaurien" (p. 49-50). La dépossession constitue un héritage virulent et la cellule familiale, profondément infectée, se désagrège définitivement au jour des noces d'or.

La description de la réunion familiale, fidèle à la logique transactionnelle du récit, commence par l'inventaire des cadeaux:

Mélanie avait apporté de la mangeaille de son restaurant pour le repas cle fête.

Emma présenta à son père une montre dorée achetée chez un marchand juif. Mélanie lui offrit une pièce d'or de $\$ 2.50$ et Rosalie donna à sa mère une demi-douzaine de cuillers à thé en simili-vermeil. (p. 46)

Quant au fils, il offre deux flacons de gin "ornés d'une étiquette d'or" (p. 46). Notons que tous les présents représentent l'or: la montre dorée, la pièce de $2.50 \$$, les cuillères en simili-vermeil, l'étiquette des flacons d'alcool. Mais cet or est inutile ou faux; la pièce de monnaie a peu de poids face à la dette de six cent dollars que Mattier ne pourra rencontrer; et la mère, recevant les cuillères, note qu'elle et son mari ne mettent jamais de sucre dans leur thé. Le gin, vite absorbé, affecte l'humeur du père qui reproche vivement à Emma, la prostituée, de ne pas lui avoir offert une montre en or véritable:

- Ben, pour c'que ça t'coûte pour le gagner l'argent, jpeux pas dire que tu t'es forcée, fit-il agressif.

- Mais s'il fallait que j'compte tout l'argent que j'vous ai donné, c'est pas ane montre, c'est ane horloge en or massif que je vous aurais apportée, répondit Emma, cinglée par cette injuste attaque. (p. 47)

Les paroles chargées de reproches et de rancune causent le départ définitif des enfants. "Leurs vies gâtées, gaspillées, aigris 
[sic] par tant de sacrifices inutiles, ils s'en allaient le cœur débordant de haine" (p. 49).

Le sacrifice originel exigé par la terre semble la source de la catastrophe. L' "amour " de la terre, thème si cher à la littérature du terroir, est une calamité - semblable à une maladie vénérienne - entraînant la haine de l'humain. Ainsi, Mélanie reproche à son père d'avoir plus aimé la terre que ses propres enfants:

- Vous avez pris note argent pis vous l'avez donné. Pourquoi? Pour rien. Vous nous avez tout arraché pour le donner, pour payer la terre et la terre est pas payée. A s'ra jamais payée. Je l'sais, moé. On aura travaillé toute note vie pour'rien, pour rien. On vous l'ôtera, vote terre, et vous finirez dans l'chemin du roi. Vous pourrez prendre ane poche et aller de porte en porte. Pis, vous finirez par crever dans l'chemin. (p. 49)

Dans cette nouvelle, la médiocrité de l'héritage paternel provoque une sinistre réaction en chaîne. L'endettement de Mattier et l'exploitation des enfants, conséquences de cet héritage, entraînent le désagrégement puis l'explosion de la cellule familiale. Les comparaisons, les métaphores et les dédoublements sécrètent un réseau sémantique imprégné du paradigme de la malédiction. Chaque enfant a un récit propre, caractérisé par la dépossession et l'exploitation, qui reflète la situation du père: l'aliénation se multiplie indéfiniment comme dans un jeu de miroirs. L'inutilité des cadeaux dorés - l'or factice des noces d'or - trahit le désastre de cinq vies et fait éclater la haine trop longtemps retenue. "Plus meurtrières que des coups de couteau" (p. 49), les paroles divisent la famille, disent toute la vérité et signent la fin de toute communication. Le père n'est plus qu'un pantin au seuil du tombeau: "D'un geste mécanique, le vieux soulevait son couteau, le mettait debout et le laissait ensuite retomber sur la table [...]" (p. 50). Et à la dernière phrase, la seule illusion qui unissait la famille, le rêve d'une maison en briques rouges, fait littéralement naufrage:

[La pluie] tombait sur le toit, elle glissait sur les fenêtres basses et c'était comme un déluge de larmes. C'était comme si la vieille maison pleurait, pleurait toutes ces vies gâtées, sur le pitoyable destin de ces êtres qu'elle avait abrité et qui, comme des épaves, s'en allaient à vau-l'eau (p. 50). 


\section{La survie d'Albert Laberge nouvelliste}

Il serait hasardeux de prévoir la réception critique de l'éventuelle réédition d'un, de plusieurs ou de tous les recueils de nouvelles de Laberge. Il est plus que probable que quelques-unes de ses nouvelles viendront nourrir des anthologies collectives - ou bien une ou plusieurs anthologies consacrées à cet auteur.

Peut-être le sadisme de Laberge envers l'ensemble de ses personnages répondait-il à son "rejet "du champ littéraire. Malgré la sérénité qu'il affichait - ou feignait d'afficher - à l'âge de la retraite, il ne pouvait vraisemblablement pas se satisfaire, ainsi qu'il le prétendait, du simple "plaisir" d'écrire. Les choix esthétiques qui ont présidé à son ceuvre ne pouvaient favoriser l'approbation de ses contemporains. Laberge le savait, et le refus de diffuser ses recueils constituait, soit un choix lucide, une façon d'éviter l'inévitable, de prévenir le scandale et la mise à l'Index, soit (voire également) la réalisation d'un désir inconscient d'autopunition; l'édition hors commerce, autocensure qui prévient ou simule la censure institutionnelle, préservait sa posture d'écrivain inconnu et, sur le plan imaginaire, d'artiste maudit. Cette malédiction, subie ou recherchée, se reflète dans la malédiction qui, d'une nouvelle à l'autre, frappe impitoyablement les personnages. La noirceur de l'univers labergien trahit-elle une "vision" pessimiste du monde, comme l'affirment de nombreux critiques, ou plutôt le transfert, sur des personnages fictifs, d'une féroce rancune contre l'institution littéraire - contre une entité castratrice profondément introjectée dans sa psyché? Ainsi, l'ouuvre de cet homme tourmenté, incapable d'assumer son désir d'être lu et reconnu comme un écrivain, d'avouer la grande frustration de sa vie, témoigne d'un conflit intérieur particulièrement violent: nous pourrions aisément voir le retour du refoulé dans la cruauté symptomatique de ses nouvelles.

Laberge maîtrise plus ou moins bien la prose littéraire ${ }^{21}$, mais il est en pleine possession de sa langue cauchemardesque, d'un alphabet morbide, macabre, dévastateur; chaque récit est une

21 Jacques Brunet, dans Albert Labenge: sa vie et son ceuvre, op. cit., consacre un chapitre à l'étude de la prose labergienne. Il affirme que Laberge est surtout à l'aise dans le langage populaire et qu'u[une] lecture attentive de son ceuvre révèle un grand nombre d'incorrections linguistiques et de grossières erreurs de style, p. 101. 
longue phrase ténébreuse dont on termine la lecture dans une sensation de soulagement. Mais - par masochisme ou par hédonisme? - on voudrait bien y plonger à nouveau, y croire encore. Laberge affichait plusieurs attributs d'un nouvelliste de génie. Mais si la majorité de ses nouvelles, prises isolément, mériteraient la réédition, leur rassemblement au sein de recueils risquerait peut-être de souffrir d'une trop grande cohérence intertextuelle. Laberge lui-même constatait que ses recueils manifestaient cette caractéristique :

Je suis sous l'impression que ce qui vous a frappé dans mes contes, est que le dénouemeni est toujours tragique, douloureux, ou d'un amer désappointement. Je vous dirai que je serais incapalble d'écrire un conte avec un dénouement heureux. Céla équivaut à dire que je veux plaire à la masse du public... or... égoìste, j'écris pour me plaire à moi-même. J'écris pour la joie d'écrire, pour la grande joie d'écrire ... ${ }^{22}$

Laberge ne voulait pas plaire par le biais de nouvelles agréables représentant des personnages sympathiques, des dénouements heureux, etc. Toutefois, l'homogénéité générale de son écriture, la surcharge complaisante dans les procédés - stylistiques et narratifs - employés pour créer un monde calamiteux, nouvelle après nouvelle, risque d'émousser l'intérêt (la fascination ou le dégoût) du lecteur qui parcourt successivement des récits trop similaires par leur noirceur. Il reste à savoir à quel point, pour le commun des lecteurs et des critiques, la troublante originalité de chaque cauchemar peut compenser ce potentiel néfaste du recueil et donner envie d'explorer davantage cet univers de damnation. Cependant, en attendant toute réédition, l'Anthologie présentée par Gérard Bessette demeure un lieu quasi obligé pour apprécier ces morceaux uniques dans le corpus nouvellier de notre littérature.

22 Albert Laberge, lettre à Maturice Lebel, cité par Gabrielle Clerc, op. cit., f. 45. 